\title{
Variation in flowering time in sorghum core collection and mapping of QTLs controlling flowering time by association analysis
}

\author{
Yousra El Mannai • Tariq Shehzad • \\ Kazutoshi Okuno
}

Received: 4 January 2011 / Accepted: 21 July 2011/Published online: 7 September 2011

(C) The Author(s) 2011. This article is published with open access at Springerlink.com

\begin{abstract}
A wide range of variation in flowering time was observed within a diversity research set of 107 sorghum accessions ranging from 56 to 133 days. Accessions were classified into early medium and late flowering groups. 45 accessions were grown under three different environments of photoperiod $(11,12$ and $15 \mathrm{~h}$ ). Sorghum accessions gradually responded to the decreasing of day-length. The $12 \mathrm{~h}$ of photoperiod could be considered as a threshold above which day-length delays the flowering time in sorghum. Association analysis was performed to identify the QTLs controlling flowering time and photoperiod sensitivity using 107 accessions of sorghum grown under natural condition and 45 accessions grown under controlled conditions. Four QTLs controlling flowering time were detected under natural condition of day-length at threshold 2.5 using $\mathrm{K}$ model. A total of seven flowering time loci were detected under controlled conditions of day-length. One QTL controlling photoperiod sensitivity was detected on chromosome 1 and one QTL controlling
\end{abstract}

Electronic supplementary material The online version of this article (doi:10.1007/s10722-011-9737-y) contains supplementary material, which is available to authorized users.

Y. El Mannai · T. Shehzad · K. Okuno $(\varangle)$

Lab of Plant Genetics and Breeding Science,

Graduate School of Life and Environmental Sciences,

University of Tsukuba, 1-1-1 Tennodai, Tsukuba,

Ibaraki 305-8572, Japan

e-mail: okusan@sakura.cc.tsukuba.ac.jp photoperiod insensitivity was detected on chromosome 4.

Keywords Flowering - LD · Photoperiod sensitivity · QTL · Sorghum

\section{Introduction}

Flowering time is one of the essential traits determining adaptation during crop domestication. In sorghum (Sorghum bicolor (L.) Moench) flowering is considered as a crucial event because of its key role in the adaptation and geographical distribution of this crop. Flowering time is affected by environmental stimuli where photoperiod is one of the major determinant factors for this trait (Kikuchi and Handa 2009). Most of plant species exhibit some degree of photoperiodism, which is a control of the time or the date of flowering by the photoperiod. Whereas the effects of photoperiod on flowering time in sorghum are essential for the crop domestication, these effects are not well understood (Michael et al. 2008). Despite extensive analysis of the day-length control of flowering in sorghum, little is known regarding effect of variation in photoperiod or day-length on flowering time of sorghum (Menz et al. 2002). Since sorghum was recognized as short-day species by Garner and Allard (1923), photoperiod sensitivity in this species has been systematically eliminated by breeders to enlarge the range of adaptability and to extend its cropping area to 
temperate environment (Chanterau et al. 2001). A better understanding of response and sensitivity of flowering time in sorghum to the photoperiod will facilitate the control of flowering time. In recent years, gene mapping using linkage disequilibrium (LD) has become one of the most active areas of research in plant genetics. LD is a powerful strategy for identifying genes underlying quantitative traits in plants (Casa et al. 2008). Thus the objectives of the current study were to analyze the variation in flowering time in a diverse core set of sorghum, to explore the sensitivity of flowering time to the variation in photoperiod and to identify QTLs controlling flowering time using association analysis.

\section{Materials and methods}

A diversity research set of 107 sorghum accessions developed by Shehezad et al. (2009a) representing African and Asian countries was used in this study (Supplementary Table 1). According to their flowering time, accessions were divided into early, medium and late flowering groups. Fifteen accessions were randomly selected from each group. A total of 45 accessions were grown as replicated sets in three identical cabinets (at the experimental field of Tsukuba University). The day-length was set to 11 , 12 and $15 \mathrm{~h}$, respectively. The main effect of the photoperiod treatments was defined for each accession by counting number of days from sowing to flowering.

Furthermore association analyses were conducted using the core collection grown under natural condition of day-length and the 45 accessions grown under controlled conditions to identify QTLs associated with flowering time and photoperiod sensitivity. A total of 98 markers previously described in Shehzad et al. (2009b) were used for association analyses. Population structure was performed using the program STRUCTURE version 2.2 (Pritchard et al. 2000). Bayesian clustering analyses with the admixture models were used where number of populations $(J)$ ranged from 2 to 9. Markov chain Monte Carlo (MCMC) sampling was repeated $1 \times 10^{6}$ times after $1 \times 10^{4}$ cycles of a burn-in period. The posterior probability of $J=2$ was the largest among other values and was selected after two times repetition. The $\mathbf{Q}$ matrix, whose $(I, j)$-th element was $q_{i j}$, was further incorporated into the association mapping models where the effect of population structure was considered. A kinship matrix, $\mathbf{K}$, was calculated as allele sharing rates of the 98 SSR markers as suggested by Zhao et al. (2007) and used in the models with $\mathrm{K}$ effect. LD between SSR markers were estimated by $D^{\prime}$ and $\boldsymbol{r}^{2}$, where $D^{\prime}$ is the standardized disequilibrium coefficient and $\boldsymbol{r}^{2}$ represents the correlation between alleles at two loci. A statistical software TASSEL (Trait Analysis by Association, Evolution and Linkage) ver.2.0.1 (Bradbury et al. 2007) was used to obtain $P$ values representing the significance of LD. To identify QTLs significantly associated with flowering time general linear model (GLM) as well as mixed linear model (MLM) were applied for analysis in TASSEL software. In GLM two different models were used (1) naïve model where there is no control of population structure and kinship (2) Q model based on population structure (Yu et al. 2006). In MLM we used two models: (1) the model which accounted for kinship (K), (2) the model that takes into account both the population structure and the kinship $(\mathrm{Q}+\mathrm{K})$.

\section{Results}

A wide range of variation in flowering time was observed within the panel of 107 sorghum accessions (Supplementary Table 1) ranging from 56 to 133 days. On the basis of number of days to flowering the 107 sorghum accessions were classified into three groups: early flowering group with less than 75 days, medium flowering from 75 to 95 days and late flowering group with more than 95 days from sowing to flowering. Under controlled conditions day-length varied substantially across experiments resulting in considerable variation in flowering time for most of the accessions from different flowering groups (Fig. 1). A photoperiod of 11 and $12 \mathrm{~h}$ accelerated flowering for the majority of early, medium and late accessions compared with $15 \mathrm{~h}$ of photoperiod. Above $12 \mathrm{~h}$ of photoperiod the increase of day-length generated a delay in flowering for accessions of all flowering groups. Difference in number of days to flowering between 12 and $15 \mathrm{~h}$ of photoperiod ranged from 4 to 18 days for the early flowering group, from 3 to 17 days in medium group and from 5 to 11 days for the late group. There was 

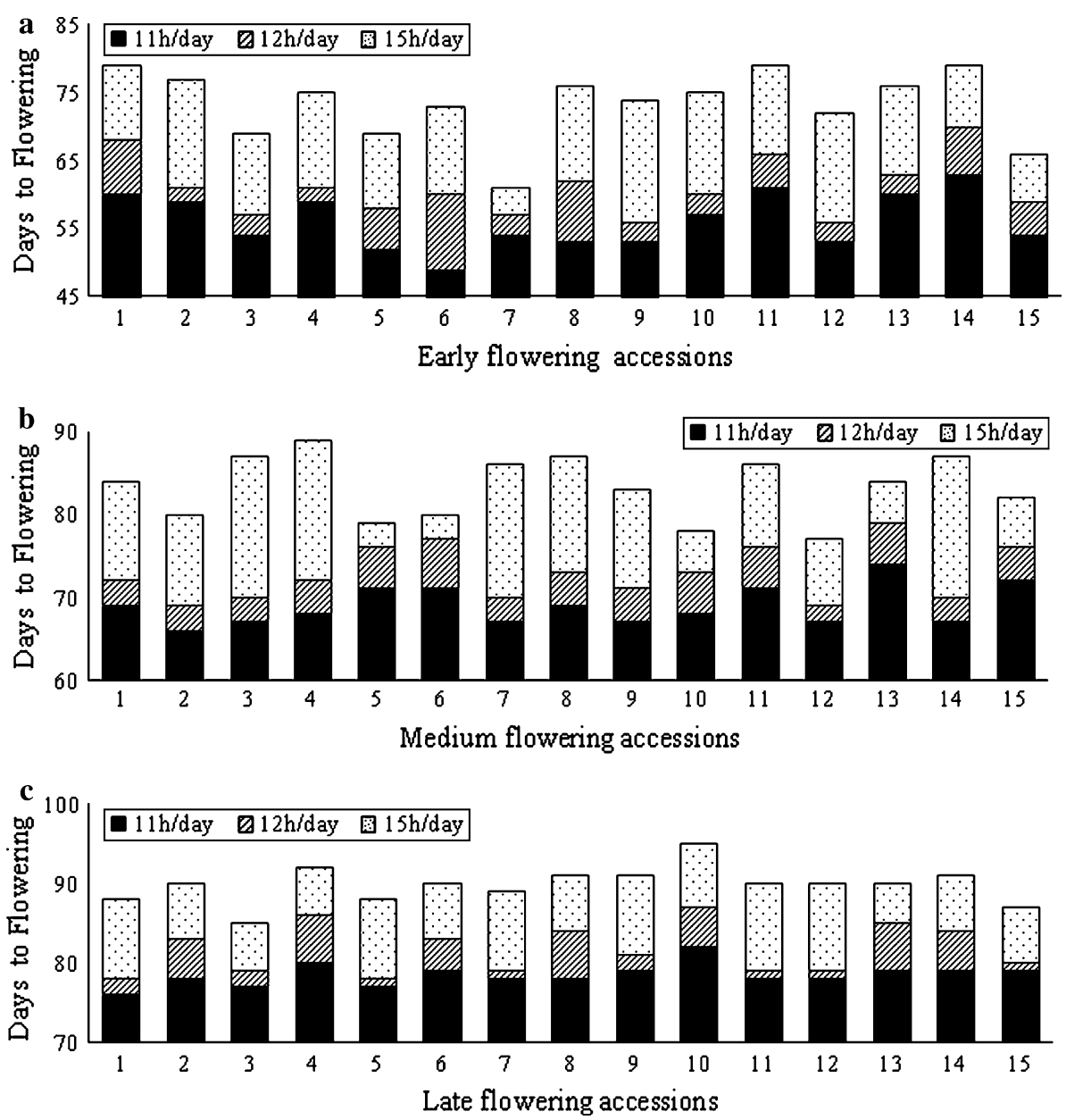

Fig. 1 Variation in flowering time in 45 sorghum accessions grown under controlled conditions of photoperiod (a early flowering accessions; b medium flowering accession; c late flowering accessions)

no gradual response of flowering time to photoperiod between 12 and $15 \mathrm{~h}$, and variation of number of days to flowering was strongly affected by the increase of the day-length. The $12 \mathrm{~h}$ of photoperiod per day could be considered as a threshold above which daylength delay the flowering time in sorghum.

Using association analysis no QTL controlling flowering time was identified under natural condition by GLM models. Using K model, four SSR loci were identified to be associated with flowering time under natural condition at a threshold of 2.5 and one locus at 2.4. Xtxp159 on chromosome 5 and Xtxp51 on chromosome 4 showed a strong association $(P<0.0001)$ with flowering time (Fig. 2a). Using naïve model three markers suggesting associations with flowering time were detected at a threshold 2. Xtxp10, was identified on chromosome 6; Xtxp159 on chromosome 5 and
Xtxp297 on chromosome 2 under 11, 12 and $15 \mathrm{~h}$ of photoperiod, respectively. Xtxp159 was detected under natural condition of day-length and short-day condition. In Q model Xtxp13 was weakly associated with flowering time. It was detected on chromosome 2 under short day conditions only. Using $\mathrm{K}$ model (Fig. 2b) four loci were identified to be associated with flowering time at a threshold 2.5. Xtxp298, Xtxp51 and Xtxp312 were detected on chromosome 2, chromosome 4 and chromosome 5 respectively, under $12 \mathrm{~h}$ of photoperiod. Xtxp100 was detected on chromosome 2 under long-day condition. Three loci were detected using $\mathrm{K}$ model at a threshold 2 under 11, 12 and $15 \mathrm{~h}$ of day-length respectively (Table 1 ). For $\mathrm{Q}+\mathrm{K}$ model (Fig 2c), the number of associated markers was the largest among all models. A total of eight markers were associated with flowering time under short-day 

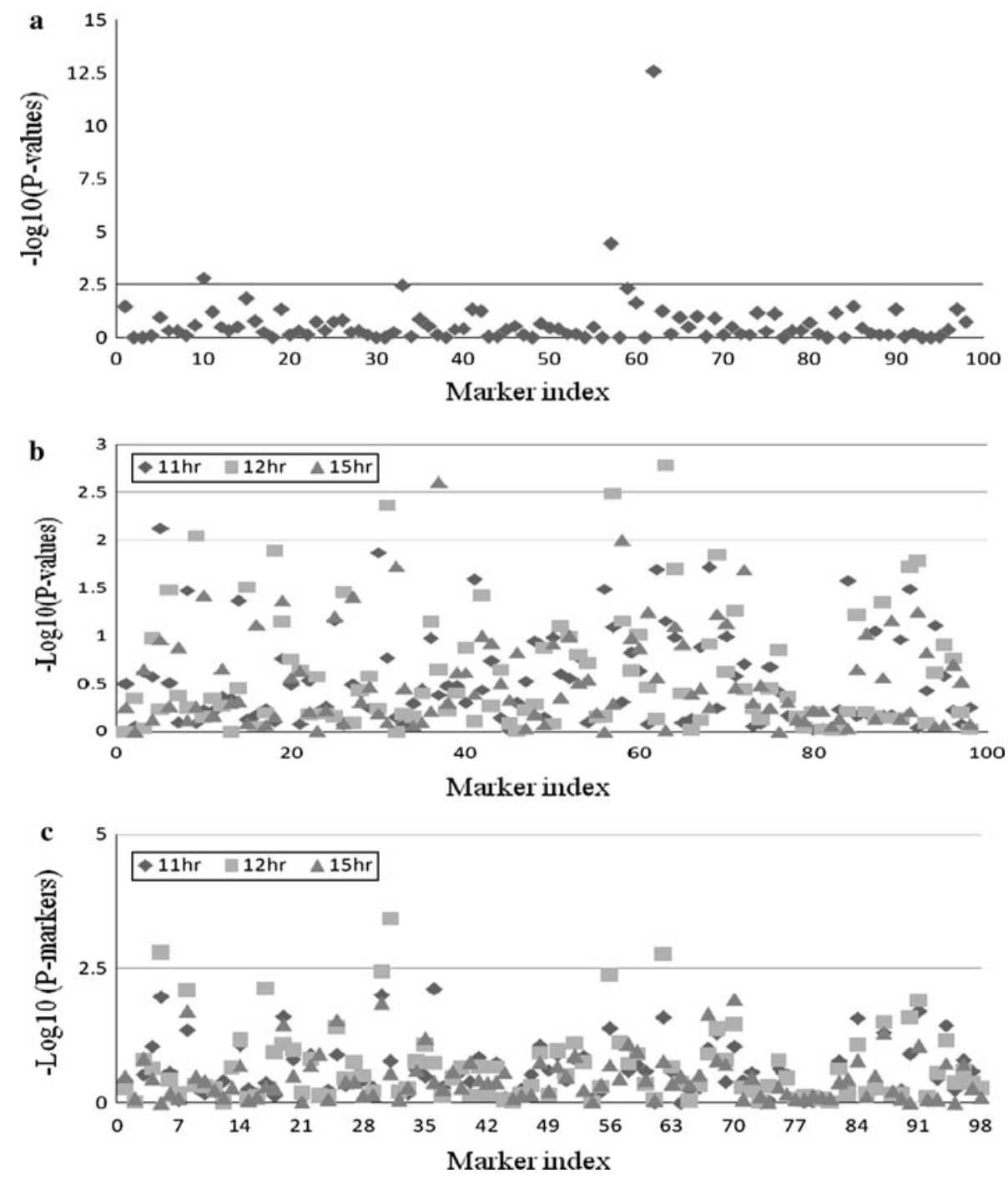

Fig. 2 a Association analysis of 98 SSR markers and flowering time using $\mathrm{K}$ model for 107 sorghum accessions under natural condition of day-length. b Association analysis of 98 SSR markers and flowering time using $\mathrm{K}$ model for 45

condition. Five loci were significantly associated with flowering time at threshold 2.5. Xtxp298, Xtxp61 and Xtxp312 on chromosome 2, chromosome 1 and chromosome 5 respectively, were fund to be the most strongly associated with flowering time under $12 \mathrm{~h}$ of photoperiod. A range of LD was observed in the 45 accessions grown under controlled conditions of photoperiod. The triangle plot for pairwise LD between marker sites in a hypothetical genome fragment, where pairwise LD values of polymorphic sites were plotted on both $\mathrm{X}$ and $\mathrm{Y}$ axis; above the sorghum accessions under controlled conditions of day-length. c Association analysis of 98 SSR markers and flowering time using $(\mathrm{Q}+\mathrm{K})$ model for 45 sorghum accessions under controlled conditions of day-length

diagonal displays $\mathrm{r} 2$ values and below the diagonal displays $P$ values from rapid 1,000 shuffle premutation test (Fig. 3). Each cell represents the relationship between two markers with the color codes indicating the significance of LD.

\section{Discussion}

The results obtained in this research suggested that sorghum accessions gradually responded to the 
Table 1 A total of loci associated with flowering time identified using 98 SSR markers

\begin{tabular}{|c|c|c|c|}
\hline Photoperiod (h) & Marker & Chromosome & $P$ values \\
\hline \multirow[t]{4}{*}{11} & Xtxp61 & 1 & 2.12317 \\
\hline & Xtxp13 & 2 & 2.05941 \\
\hline & Xtxp315 & 2 & 2.11415 \\
\hline & Xtxp10 & 6 & 2.11991 \\
\hline \multirow[t]{13}{*}{12} & Xtxp61 & 1 & 2.78197 \\
\hline & Xtxp75 & 1 & 2.04416 \\
\hline & Xtxp279 & 1 & 2.08766 \\
\hline & Xtxp302 & 1 & 2.11431 \\
\hline & Xtxp13 & 2 & 2.43607 \\
\hline & Xtxp13 & 2 & 2.26574 \\
\hline & Xtxp298 & 2 & 3.42966 \\
\hline & Xtxp298 & 2 & 2.36285 \\
\hline & Xtxp51 & 4 & 2.49049 \\
\hline & Xtxp212 & 4 & 2.39630 \\
\hline & Xtxp159 & 5 & 2.12045 \\
\hline & Xtxp159 & 5 & 2.75682 \\
\hline & Xtxp312 & 5 & 2.78336 \\
\hline \multirow[t]{3}{*}{15} & Xtxp297 & 1 & 2.02636 \\
\hline & Xtxp100 & 2 & 2.60788 \\
\hline & Xtxp27 & 4 & 2.00007 \\
\hline \multirow[t]{5}{*}{ Natural condition } & Xtxp58 & 1 & 2.79588 \\
\hline & Xtxp56 & 2 & 2.48148 \\
\hline & Xtxp51 & 4 & 4.43995 \\
\hline & Xtxp59 & 4 & 2.35654 \\
\hline & Xtxp159 & 5 & 12.59246 \\
\hline
\end{tabular}

decreasing of day-length. Long-day conditions delay the flowering time by increasing the number of days to flowering. These results were confirmed previously by Garner and Allard (1923) and by Folliard et al. (2004) who proved that for sorghum crop, progress towards flowering is accelerated when day-length decreases. On the basis of these outcomes we suggested that the exacted photoperiod compulsory for sorghum flowering belongs to the interval of 11-12 $\mathrm{h}$ of photoperiod. Variation in response to photoperiod and sensitivity to it fluctuated within accessions. Some accessions seemed to be weakly affected by change in photoperiod. Other accessions appeared to be strongly affected by changes in daylength and subsequently severely sensitive to photoperiod. Kassam and Andrews (1975) suggested that there were two major mechanisms controlling flowering time and adaptation in sorghums; firstly mechanism in which genotypes are sensitive or insensitive to photoperiod and secondly mechanism in which genotypes are inherently early/late flowering.

The association analysis was performed to identify QTLs controlling flowering time of the genotyping data of 98 SSR markers. Using core collection grown under natural condition of day-length Xtxp51 and Xtxp159 were fund to be significantly associated with flowering time by K model. Same loci were identified under $12 \mathrm{~h}$ of photoperiod. These loci were detectable under varying photoperiod indicating that their expression is photoperiod insensitive. Two loci controlling flowering time were located-Xtxp61 on chromosome 1 and Xtxp13 on chromosome 2 were expressed exclusively in short-day conditions suggesting that their expression was relatively sensitive to photoperiod. These two loci accelerated flowering under short photoperiod. We also detected two photoperiod sensitive QTLs on chromosome 2 and chromosome 6 since they were only detectable under $11 \mathrm{~h}$ of photoperiod suggesting that there is a minimum photoperiod necessary for their expression. These loci are sensitive to photoperiod of some degree. Three loci were detectable exclusively under long-day condition, suggesting that there is a maximum photoperiod necessary for their expression (Table 1).

We have compared our results with other studies of the photoperiod response in sorghum to account for possible orthologies. The QTL detected on chromosome 6 in this study has a close map position to the one detected by Lin et al. (1995) and Chanterau et al. (2001). This QTL was detected under $11 \mathrm{~h}$ of photoperiod and appeared to be responsible for the control of the photoperiod sensitivity in sorghum. In same way QTL detected on chromosome 5 has the same position as previously reported by Srinivas et al. (2009) and can be considered as QTL controlling photoperiod sensitivity in sorghum. The QTL detected on chromosome 1 only under short daylength appears to be newly mapped in sorghum. No previous study reported QTL controlling flowering time and sensitivity to photoperiod located in same position as the one detected on chromosome 1 in this study. This QTL can be considered as a QTL controlling sensitivity to photoperiod in sorghum. Moreover the QTL detected on chromosome 4 under 
Fig. 3 LD plot generated by 98 SSR markers. Each cell represents the relationship between two markers with the color codes for the presence of significant LD. Colored bar codes for the significant threshold levels

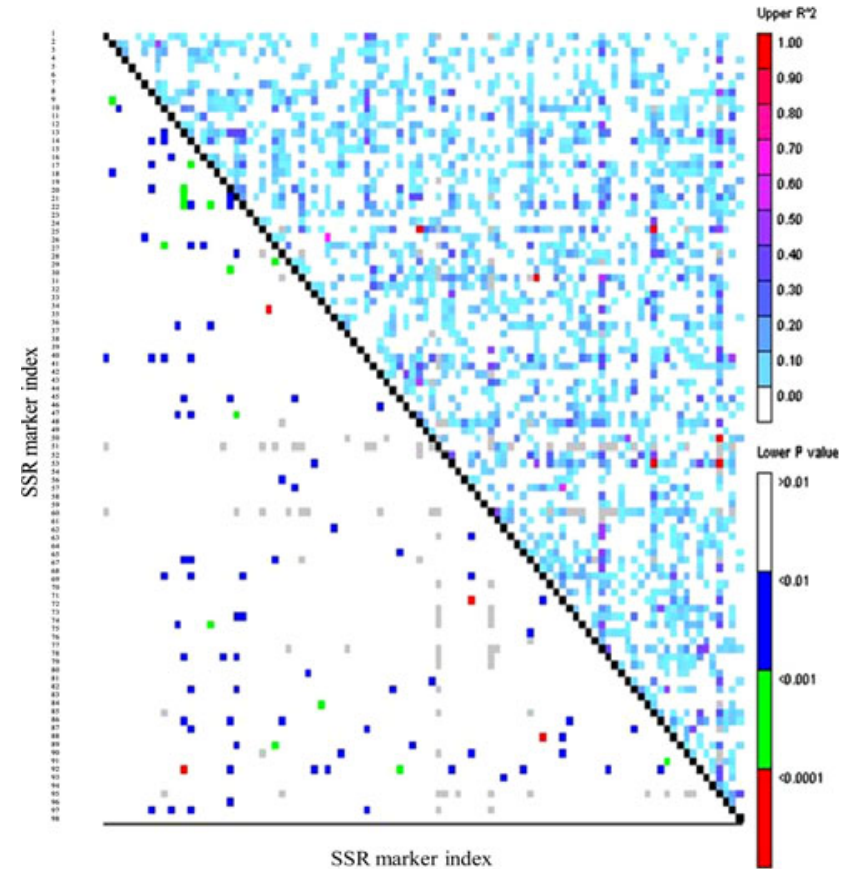

detected in this study. Further studies are required to elucidate the expression of these QTLs and investigate their effects on flowering time in sorghum.

Open Access This article is distributed under the terms of the Creative Commons Attribution Noncommercial License which permits any noncommercial use, distribution, and reproduction in any medium, provided the original author(s) and source are credited.

\section{References}

Bradbury PJ, Zhang Z, Kron DE, Casstevens TM, Ramdoss Y, Buckler ES (2007) TASSEL: Software for association mapping of complex traits in diverse samples. Bioinformatics 23:2633-2635

Casa AM, Pressoir G, Brown PJ, Mitchell SE, Rooney WL, Tuinstra MR, Franks CD, Kresovich S (2008) Community resources and strategies for association mapping in sorghum. Crop Sci 48:30-40

Chanterau J, Trouche G, Rami JF, Deu M, Barro C, Grivet L (2001) RFLP mapping of QTLs for photoperiod response in tropical sorghum. Euphytica 120:183-194

Folliard A, Traore PCS, Vaksmann M, Kouressy M (2004) Modeling of sorghum response to photoperiod: a threshold-hyperbolic approach. Field Crop Res 89:59-70

Garner WW, Allard HA (1923) Further studies in photoperiodism, the response of the plant to relative length of day and night. J Agric Res 23:871-920 
Kassam AH, Andrews DJ (1975) Effect of sowing date on growth development and yield photosensitive sorghum at Samaru northen Nigeria. Exp Agric 11:227-240

Kikuchi R, Handa H (2009) Photoperiodic control of flowering in barley. Breed Sci 59:546-552

Lin Y-R, Schertz KF, Paterson AH (1995) Comparative analysis of QTLs affecting plant height and maturity across the Poaceae, in reference to an inter-specific sorghum population. Genetics 141:391-411

Menz MA, Klein RR, Mullet JE, Obert JA, Unruh NC, Klein PE (2002) A high-density genetic map of Sorghum bicolor (L.) Moench based on 2926 AFLP, RFLP and SSR markers. Plant Mol Biol 48:483-499

Michael D, Mamoutou K, Michel V, Benoit C, Jaques C (2008) A model of sorghum photoperiodism using the concept of threshold-lowering during prolonged appetence. Europ J Agro 28:74-89

Pritchard JK, Stephens M, Rosenberg NA, Donnelly P (2000) Association mapping in structured populations. Am J Hum Genet 67:170-181

Shehzad T, Okuizumi H, Kawase M, Okuno K (2009a) Development of SSR based sorghum (Sorghum bicolor (L.) Moench) diversity research set and its evaluation by morphological traits. Genet Resour Crop Evol 56:809-827
Shehezad T, Iwata H, Okuno K (2009b) Genome-wide association maping of quantitative traits in sorghum (Sorghum bicolor (L.) Moench) by using multiple models. Breed Sci 59:217-227

Srinivas G, Satish K, Madhusudhana R, Nagaraja RR, Murali SM, Seetharama N (2009) Identification of quantitative trait loci for agronomically important trait and their association with genic-microsatellite markers in sorghum. Theor Appl Genet 118:1439-1454

Stich B, Albrech E, Frish MM, Maurer HP, Heckenberger M, Reif JC (2005) Linkage disequilibrium in European elite maize germplasm investigated with SSRs. Theo Appl Genet 111:723-730

Yu J, Pressoir G, Briggs WH, VrohBi I, Yamasaki M, Doebley JF, McMullen MD, Gaut BS, Nielsen DM, Holland JB, Kresovich S, Buckler ES (2006) A unified mixed-model method for association mapping that accounts for multiple levels of relatedness. Nat Genet 38:203-208

Zhao K, Aranzana MJ, Kim S, Lister C, Shindo C, Tang C, Toomajian C, Zheng H, Dean C, Marjoram P, Nordborg M (2007) An Arabidopsis example of association mapping in structured samples. PLOS Gene 3:e4 pp 71-81 